\title{
Status of the T2K experiment
}

\author{
Ken SAKASHITA ${ }^{* \dagger}$ \\ HIGH ENERGY ACCELERATOR RESEARCH ORGANIZATION, KEK \\ E-mail: kenshepost.kek.jp
}

T2K (Tokai-to-Kamioka) is a second generation long baseline neutrino experiment utilizing a newly built neutrino source with a MW class high energy proton accelerator complex (J-PARC neutrino facility), a near neutrino detector (ND280) to characterize the neutrino beam 280 meters from the source, and Super-Kamiokande as the far detector at $295 \mathrm{~km}$. The primary motivation for $\mathrm{T} 2 \mathrm{~K}$ is the discovery of the $v_{\mu}$ to $v_{e}$ conversion phenomena and, as a consequence, the finite value of the $\theta_{13}$ mixing angle. It will also conduct a precise measurement of $\theta_{23}$ and the mass difference of neutrino mass eigenstate. The ultimate goal for $\mathrm{T} 2 \mathrm{~K}$ is to establish the lepton flavor mixing structure. Construction of the J-PARC neutrino facility was completed in March 2009 and engineering operation of the $\mathrm{T} 2 \mathrm{~K}$ started as scheduled the following month. We report a general introduction to $\mathrm{T} 2 \mathrm{~K}$, and present the current beam commissioning status and the status of preparations towards the start of the experiment in fall 2009.

European Physical Society Europhysics Conference on High Energy Physics

July 16-22, 2009

Krakow, Poland

* Speaker.

${ }^{\dagger}$ On behalf of the T2K collaboration 


\section{Introduction}

Neutrino oscillation is a unique tool to study neutrino mass and mixing as well as a probe of new physics beyond the Standard Model (SM). Past experimental results of the neutrino oscillation indicate that neutrinos have mass and structure of the mixing matrix is completely different than the quark mixing matrix. These results are the first observation indicating physics beyond the SM.

Next step of neutrino oscillation experiment is to discover the last unknown mixing angle $\left(\theta_{13}\right)$ and the leptonic $\mathrm{CP}$ violating phase. In particular, determination of the size of $\theta_{13}$ is important in order to determine the direction of future neutrino experiments; for instance, a sufficiently large $\theta_{13}$ enable us to measure the leptonic $\mathrm{CP}$ violation phase in future experiments using a conventional neutrino beam.

T2K experiment aims for discovery of a finite $\theta_{13}$ by observing $v_{e}$ appearance $\left(v_{\mu} \rightarrow v_{e}\right.$ oscillation), and precise measurement of oscillation parameters, $\Delta m_{23}^{2}$ and $\sin ^{2}\left(2 \theta_{23}\right)$, in $v_{\mu}$ disappearance.

\section{T2K experiment}

T2K experiment is the accelerator-base long baseline neutrino oscillation experiment in Japan. Muon neutrinos $\left(v_{\mu}\right.$ 's) are produced by striking protons from proton synchrotron in J-PARC on a graphite target. Neutrino beam is directed to the Super-Kamiokande detector located at $295 \mathrm{~km}$ from the target.

One of main features in $\mathrm{T} 2 \mathrm{~K}$ experiment in order to enhance the physics sensitivity is intense and narrow-band neutrino beam. We employ "off-axis" method[1] in order to produce the intense narrow-band neutrino beam. The beam energy is determined by selecting the angle between the direction of parent $\pi^{+}$and neutrinos. We set the angle to $2.5^{\circ}$ in order to tune the beam energy to be $0.6-0.7 \mathrm{GeV}$ which corresponds to the current value of $\Delta m_{23}^{2}$ with the baseline of $295 \mathrm{~km}$. The number of neutrino interactions in SK detector is expected to be $\sim 1200$ Charged Current interactions per year. It is two times larger than the case of "on-axis" beam. Moreover, the offaxis beam reduces the high energy tail and it enables us to reduce the background events for $v_{e}$ appearance and $v_{\mu}$ disappearance.

Second feature is far detector, Super-Kamiokande (SK) water cherenkov detector because of its good particle identification and energy resolution. It composes water of $50 \mathrm{kton}$ in total $(22.5 \mathrm{kton}$ fiducial). We are able to identify particle using the features of the Cherenkov ring; shower-type ring for electron while sharp-edged ring for muon. The particle separation is better than $99 \%$ at T2K beam energy. The uncertainty of the energy scale is $\sim 2 \%$.

Third feature is that we are able to reconstruct the energy of neutrino by measuring momentum of charged lepton and assuming the Charged Current Quasi-elestic (CCQE) interaction. CCQE interactions dominate at $\mathrm{T} 2 \mathrm{~K}$ beam energy.

We measure $v_{e}$ appearance signal by detecting single electron from $v_{e}$ CCQE interaction in SK detector. We expect that our sensitivity of $\theta_{13}$ is $\sin ^{2}\left(2 \theta_{13}\right)<0.006$ at $90 \%$ C.L. for $\delta=0$, $\Delta m_{13}^{2}=2.5 \times 10^{-3} \mathrm{eV}^{2}$ (normal hierarchy) and $\sin ^{2}\left(2 \theta_{23}\right)=1$ with $8 \times 10^{21}$ protons on the target (p.o.t.) measurement. It is more than a order of magnitude improvement from the current world limit of $\sin ^{2}\left(2 \theta_{13}\right)$ [2]. 
We measure $v_{\mu}$ disappearance as both a suppression in the total number of $v_{\mu}$ events observed at SK and a distortion of the energy spectrum compared to the expected energy spectrum from the production point. We expect that our sensitivities are $\delta\left(\Delta m_{23}^{2}\right)<1 \times 10^{-4} \mathrm{eV}^{2}$ and $\delta\left(\sin ^{2}\left(2 \theta_{23}\right)\right) \sim$ $1 \%$ with $8 \times 10^{21}$ p.o.t. measurement.

\section{T2K Beam line commissioning}

T2K neutrino beam-line consists of a $30 \mathrm{GeV}$ proton synchrotron (J-PARC MR), a primary proton transportation line, a hadron production target ( $90 \mathrm{~cm}$ long), a set of focusing horn magnets for secondary particles, a decay volume, and a beam dump. Protons are accelerated by MR to a kinetic energy of $30 \mathrm{GeV}$. After acceleration, all protons are extracted in a single turn to the neutrino beam-line. The beam is bent by $90^{\circ}$ toward the direction of SK by a set of super conduction magnets and normal conduction magnets. Produced pions at the target were focused by three horn magnets and decay into muons and $\nu_{\mu}$ 's in the decay volume.

Construction of T2K neutrino beam-line has been completed in March 2009 after 5 years construction. Only first horn magnet was installed at that time because we found interference between horn magnet and a helium vessel of the target area for second and third horn magnets. We monitor the neutrino beam direction by measuring secondary muon beam profile every spill in order to keep the beam direction stable within $1 \mathrm{mrad}$ so that to keep uncertainty of oscillation parameter small.

Engineering operation (beam commissioning) of neutrino beam-line has started in April 2009 as scheduled. The beam intensity was low in order to reduce radio-activtion of target area for second and third horn installation after the commissioning. Typical beam intensity was $4 \times 10^{11}$ protons per pulse, which is $0.1 \%$ of the design beam intensity.

Muon signal was observed in the first shot just after turning on all the magnets in the primary beam-line. It indicates that we successfully produced neutrino beam. After we tuned each magnet in the primary beam-line with 9 beam shots, the proton beam hit around the center of the target (Fig. 11-left).

In the commissioning, all the beam-line equipments were successfully work as expected. Super conduction combined function magnets also worked as expected performance. We were able to transfer the beam without any significant beam loss. Beam position on the target was well controlled. Proton beam was tuned within $3 \mathrm{~mm}$ from design orbit. Moreover, increase in muon yield after turning on the horn magnets was observed by muon monitor (Fig. 1-right). Proton beam intensity $(I)$ and muon's direction $\left(\theta_{\mu}\right)$ was stable, $\delta I<1 \%$ and $\delta \theta_{\mu}<1 \mathrm{mrad}$, respectively, during 0.5 hour contiguous operation. Beam monitor and online data acquisition system were stable running during the beam commissioning.

\section{Prospects}

Second and third horns were successfully installed in this summer. Construction of near neutrino detector system is going on. One of main neutrino detector, on-axis neutrino beam direction monitor (INGRID), was also successfully installed. Other neutrino detectors, off-axis neutrino 

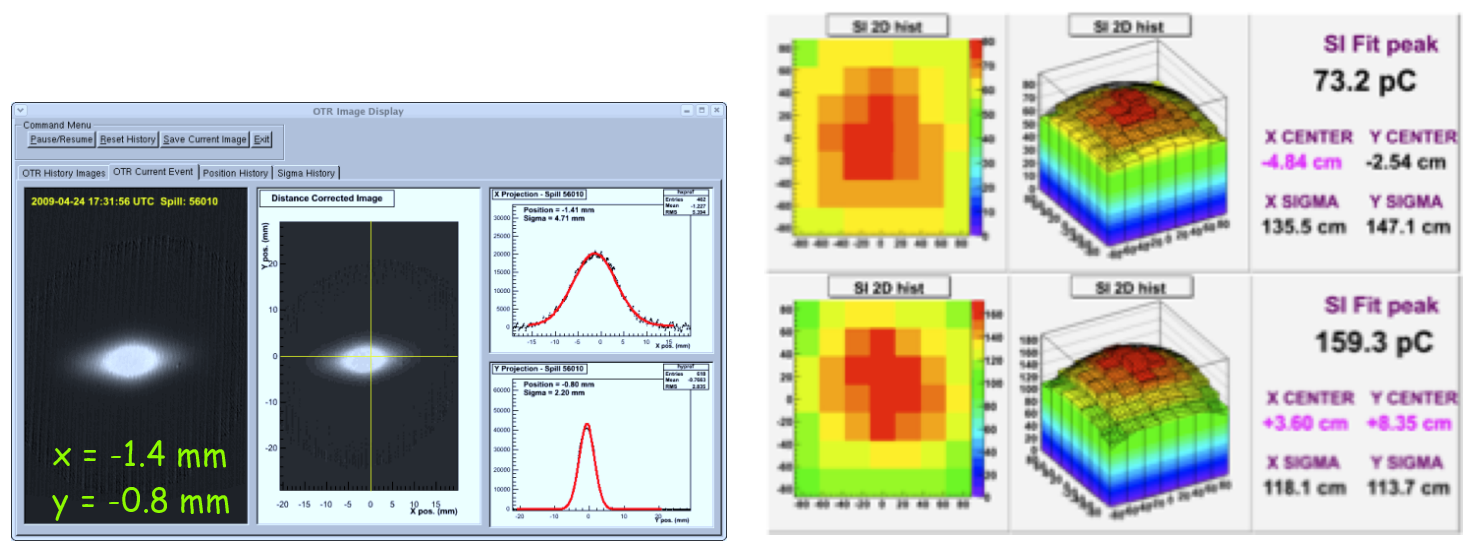

Figure 1: (Left plot) Proton beam signal by fluorescence plate which placed in front of the target after we tuned the magnets in primary beam-lines with 9 shots beam. Beam was hit around the center of target. (Right plot) Muon profile by muon monitor which placed downstream the beam dump. Top (bottom) figure shows the muon profile and yield with horn off (on).

detector complex, was under construction. All the detector installation will be completed until December 2009.

We aim for better sensitivity than the current world limit [2] in 2010 as a first step. T2K beam commissioning will restart from October with high intense beam and full setup of horn magnets. Next step, we hope to discover $v_{e}$ appearance with $1 \sim 2 \times 10^{7} \mathrm{MW} \cdot \mathrm{sec}$. in a few year. Moreover, we aim for making final results with $3.75 \times 10^{7} \mathrm{MW} \cdot \sec \left(8 \times 10^{21}\right.$ protons on the target).

\section{Summary}

T2K experiment aims for a discovery of $\theta_{13}$ and precise measurement of $\Delta m_{23}^{2}$ and $\sin ^{2}\left(2 \theta_{23}\right)$ by using a narrow-band intense $v_{\mu}$ beam and Super-Kamiokande detector. T2K has a physics potential that possible to measure $\sin ^{2}\left(2 \theta_{13}\right)$ less than 0.006 with $90 \%$ C.L. and to measure $\delta\left(\Delta m_{23}^{2}\right)<1 \times 10^{-4} \mathrm{eV}^{2}$ and $\delta\left(\sin ^{2}\left(2 \theta_{23}\right)\right) \sim 1 \%$ with $8 \times 10^{21}$ p.o.t. measurement.

First beam commissioning was completed in April and May 2009. Neutrino production was confirmed with observing muon signal. We also confirmed basic functionality of the T2K beamline. The beam commissioning will restart from October 2009 after installation of full horn magnet system and one of main neutrino detector. We aim for better sensitivity than the current world limit in 2010 .

\section{References}

[1] D. Beavis, A. Carroll, I. Chiang, et al., Proposal of BNL AGS E-889 (1995).

[2] M. Apollonio et al. [CHOOZ Collaboration], Eur. Phys. J. C 27, 331 (2003). 